Research article

\title{
Comparative adventitious root development in pre-etiolated and flooded Arabidopsis hypocotyls exposed to different auxins
}

\author{
Cibele Tesser da Costa ${ }^{\mathrm{a}}$, Marcos Letaif Gaeta ${ }^{\mathrm{b}}$, Jorge Ernesto de Araujo Mariath ${ }^{\mathrm{b}}$, \\ Remko Offringa ${ }^{c}$, Arthur Germano Fett-Neto ${ }^{a, *}$ \\ a Plant Physiology Laboratory, Center for Biotechnology, Federal University of Rio Grande do Sul (UFRGS), CP 15005, Porto Alegre, RS, 91501-970, Brazil \\ ${ }^{\mathrm{b}}$ Plant Anatomy Laboratory, Department of Botany, Federal University of Rio Grande do Sul (UFRGS), Av. Bento Gonçalves 9500, Porto Alegre, RS, 91501-970, Brazil \\ ${ }^{\mathrm{c}}$ Department of Molecular and Developmental Genetics, Institute Biology Leiden, Sylvius Laboratory, Sylviusweg 72, 2333 CB Leiden, The Netherlands
}

\section{A R T I C L E IN F O}

\section{Keywords:}

Adventitious rooting

Auxin

Arabidopsis

Auxin-related genes

Gene expression

\begin{abstract}
A B S T R A C T
Adventitious roots (ARs) emerge from stems, leaves or hypocotyls, being strategic for clonal propagation. ARs may develop spontaneously, upon environmental stress or hormonal treatment. Auxins strongly influence AR development (ARD), depending on concentration and kind. However, the role of different types of auxin is rarely compared at the molecular level. Rooting triggered by light exposure and flooding was examined in intact etiolated Arabidopsis thaliana hypocotyls treated with distinct auxin types. Morphological aspects, rooting-related gene expression profiles, and IAA immunolocalization were recorded. NAA and 2,4-D effects were highly dose-dependent; at higher concentrations NAA inhibited root growth and 2,4-D promoted callus formation. NAA yielded the highest number of roots, but inhibited elongation. IAA increased the number of roots with less interference in elongation, yielding the best overall rooting response. IAA was localized close to the tissues of root origin. Auxin stimulated ARD was marked by increased expression of PIN1 and GH3.3. NAA treatment induced expression of CYCB1, GH3.6 and ARF8. These NAA-specific responses may be associated with the development of numerous shorter roots. In contrast, expression of the auxin action inhibitor IAA28 was induced by IAA. Increased PIN1 expression indicated the relevance of auxin efflux transport for focusing in target cells, whereas GH3.3 suggested tight control of auxin homeostasis. IAA28 increased expression during IAA-induced ARD differs from what was previously reported for lateral root development, pointing to yet another possible difference in the molecular programs of these two developmental processes.
\end{abstract}

\section{Introduction}

Adventitious roots (ARs) normally emerge from the stem, leaves or, occasionally, the pericycle of hypocotyls or older roots and may develop spontaneously from stem cells or be stimulated under environmental stress. ARs are strategic for forestry and horticulture, especially when commercial materials are clonal. These roots develop in three phases: induction, initiation and expression, and most phytohormones have been shown to participate in different steps of AR development (reviewed by Da Costa et al., 2013). Although the endogenous auxin indole-3-acetic acid (IAA) is able to stimulate ARs, another endogenous auxin, indole-3-butyric acid (IBA), often promotes rooting more efficiently than IAA, being preferred in clonal propagation of commercial species (De Klerk et al., 1999). IBA is also thought to be a transport intermediate of IAA (Strader et al., 2010). Naphthalene acetic acid (NAA) is a synthetic form of auxin which promotes rooting and has higher metabolic stability (De Klerk et al., 1999). Different auxin types were investigated in $A$. thaliana inflorescence segments and IAA, IBA and NAA induced ARs formation, but 2,4-dichlorophenoxyacetic acid (2,4-D) and picloram induced callus formation at the same concentration (Verstraeten et al., 2013).

TRANSPORT INHIBITOR RESPONSE 1 (TIR1) and AUXIN RECEPTOR F-BOX PROTEINS (AFB) family of F-box proteins act as auxin receptors (Dharmasiri et al., 2005). Auxin promotes recruitment of the Aux/IAA transcriptional repressors, such as the lateral root inhibitor IAA28 (Verstraeten et al., 2014), by the SCF ${ }^{\mathrm{TIR} 1 / \mathrm{AFB}}$ (Skp1/Cullin/F-

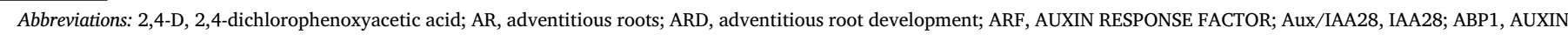

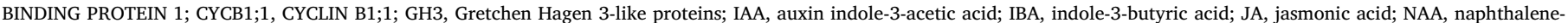

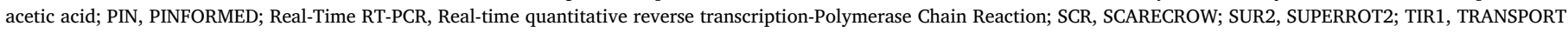
INHIBITOR RESPONSE 1

* Corresponding author.

E-mail address: fettneto@cbiot.ufrgs.br (A.G. Fett-Neto).
} 
box) E3 ubiquitin ligase complex, leading to their subsequent ubiquitination and degradation (Dharmasiri et al., 2005; Maraschin et al., 2009). This releases the Auxin Response Factors (ARFs) from the repressive action of Aux/IAAs, allowing the transcription of auxin responsive genes (Maraschin et al., 2009). Besides the nuclear TIR1/AFB auxin receptors, AUXIN BINDING PROTEIN 1 (ABP1) has been identified as an extracellular auxin receptor, displaying high specific affinity. ABP1 signaling includes activation of ATPase proton pumps, apoplast acidification, and input $\mathrm{K}^{+}$channels, as well as regulation of auxin transporters (reviewed by Da Costa et al., 2013). However, recent independent findings identified null alleles of ABP1 that develop like wild type plants under normal growth conditions (Strader and Zhao, 2016).

Members of the Gretchen Hagen 3-like (GH3) protein family are involved in auxin homeostasis, encoding IAA-amido synthetases which conjugate physiologically active excess free IAA with amino acids (Kramer and Ackelsberg, 2015). Jasmonic acid (JA) homeostasis is also modulated by the auxin-inducible GH3.3, GH3.5 and GH3.6 genes, finetuning AR initiation (Gutierrez et al., 2012). ARF6 and ARF8 are positive regulators and ARF17 is a negative regulator of these three GH3 genes which control JA conjugation with amino acids, thereby downregulating the rooting inhibitor COI1 signaling pathway (Gutierrez et al., 2012). ARF17 expression is controlled by AGO1, which acts in the crosstalk between auxin and light in adventitious rooting (Sorin et al., 2005). SUPERROT2 (SUR2) gene, encoding CYP83B1 protein, a cytochrome P450-dependent monooxygenase, modulates IAA and glucosinolate biosynthesis in Arabidopsis (Bak et al., 2001).

In shoots auxin moves basipetally, whereas in roots its transport occurs in both directions (Morris et al., 2010). Auxin uptake by cells is facilitated by influx transporters called AUXIN1/LIKE AUXIN1 (AUX1/ LAX1) (Péret et al., 2012). Polar auxin transport (PAT) is mediated by efflux transporter proteins and the most important for auxin polar transport are the PINFORMED (PIN) auxin efflux carriers. The auxin efflux function of PIN proteins was clearly shown both in plants and heterologous systems (reviewed by Nodzyński et al., 2012). Another group of auxin transporters includes ATP-binding cassette (ABC) transporters (Kamimoto et al., 2012), among which the P-glycoprotein (PGP)/ABCB subclass is relevant for auxin transport both at cellular and long-distance levels (reviewed by Geisler and Murphy, 2006, Kamimoto et al., 2012). Auxin transport may be regulated by ABP1, which functions to promote clathrin-mediated endocytosis of PIN auxin efflux carriers. Binding of auxin inhibits ABP1 action, thereby stabilizing PINs at the plasma membrane, causing auxin to enhance its own efflux (reviewed by Da Costa et al., 2013).

Establishment of the root meristem and the control of cell division, differentiation and cell homeostasis involve transcriptional factors of the GRAS family, including SCARECROW (SCR) and SHORTROOT (SHR) (reviewed by Drisch and Stahl, 2015). Expression of SCR is necessary for quiescent center identity and to maintain the surrounding stem cells (reviewed by Drisch and Stahl, 2015). B-type cyclins play roles in the regulation of mitotic and meiotic cell cycles (Pérez-Hidalgo et al., 2008). CYCB1;1 has been used as a marker gene to monitor cell mitotic activity along the root initiation process through Real Time PCR analysis or fusion with reporter systems (Himanen et al., 2002; Yin et al., 2014).
Despite the importance of auxin in adventitious rooting, knowledge about plant responses at the molecular level and the role of different auxin types is still limited. The present work addresses the question whether different natural and synthetic auxins, which differ in metabolic stability, homeostasis and transport, trigger similar gene expression profiles in relation to ARD responses in pre-etiolated and flooded Arabidopsis hypocotyls, compared to rooting in absence of exogenous auxin. Moreover, auxin distribution during AR formation was visualized through IAA immunolocalization.

\section{Materials and methods}

\subsection{Plant material and growth conditions}

Arabidopsis thaliana seeds were obtained from ABRC - The Arabidopsis Biological Resource Center (Ohio, USA) and the ecotype Col-0 was used in all experiments. Seed sterilization was carried out according to Correa et al. (2012). Surface sterilized seeds of Col-0 were placed on medium $0.1 \times$ MS supplemented with $2 \%$ sucrose and solidified with agar $(0.6 \% \mathrm{w} / \mathrm{v})$ in 24 well microplates of $2 \mathrm{~mL}$ each. The plates were wrapped with aluminum foil and maintained in the dark for 5 days at $20 \pm 2{ }^{\circ} \mathrm{C}$ to allow for germination and etiolation. Subsequently, $0.5 \mathrm{~mL}$ of liquid $0.3 \%$ agar medium with or without auxins was aseptically added into each well, covering part of the hypocotyl (Correa et al., 2012). All media $\mathrm{pH}$ were adjusted to 5.8 prior to autoclave sterilization. Seedlings were then transferred to light, with a photoperiod of $16 \mathrm{~h}$ per day and $45 \mu \mathrm{mol} \mathrm{m}^{-2} \mathrm{~s}^{-1}$ of photosynthetically active radiation (PAR).

\subsection{Auxin treatments}

To evaluate the influence of different auxin types and concentrations in adventitious rooting, etiolated seedlings were treated with $57 \mu \mathrm{M}$ of IAA (previously described by Correa et al., 2012); 2, 10, 40 or $57 \mu \mathrm{M}$ of NAA or $500 \mathrm{nM}, 1,2$ or $5 \mu \mathrm{M}$ of 2,4-D. Stocks were freshly prepared, dissolved in $\mathrm{NaOH} 10 \mathrm{mM}$, diluted in water and added to the media at indicated concentrations before autoclaving. To access whether autoclaving auxins could yield different responses compared to filter sterilization of these phytohormones, the number of adventitious roots and the root length of the longest adventitious root were compared by treating with auxins sterilized by each one of the methods. No difference was observed for the parameters monitored (see Supplementary Fig. 1). All media pH were adjusted to 5.8 prior to autoclave sterilization. The ARs were counted and root length of the longest root was measured four and eight days after treatment with auxins and transfer to light (Fig. 1).

\subsection{Immunohistological analysis}

Etiolated seedlings were treated with $57 \mu \mathrm{M}$ of IAA or with media devoid of auxins and monitored for AR development. Seedling samples were harvested at the end of etiolation period (day zero) and at two and four days after exposure of seedlings to light and auxins (Fig. 1). The samples were fixed in $4 \%$ formaldehyde $[\mathrm{w} / \mathrm{v}]$ in $0.1 \mathrm{M}$ sodium

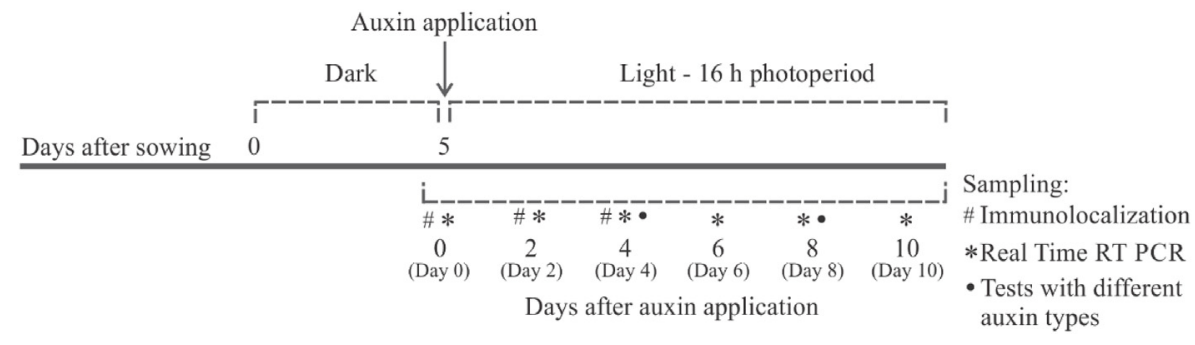

Fig. 1. Scheme of the pre-etiolated + flooding seedling system for rooting, indicating sampling time points. 
phosphate buffer $\mathrm{pH} 7.2$ at $4^{\circ} \mathrm{C}$. After $24 \mathrm{~h}$, they were washed three times with sodium phosphate buffer $\mathrm{pH} 7.2$ and kept in this solution for $48 \mathrm{~h}$. The seedlings were then dehydrated in an ethanol series, placed in gelatin capsules, embedded in resin (LR white resin - Sigma Aldrich) and polymerized at $50{ }^{\circ} \mathrm{C}$ for $24 \mathrm{~h}$. Samples were transversally sectioned in a microtome with glass blades $(2 \mu \mathrm{m}$ thick) and placed in slides covered with gelatin. The antibody incubation was done following Sakata et al. (2010), using the anti-IAA monoclonal antibody (Sigma-Aldrich, St Louis, USA) and Alexa Fluor 488-conjugated goat anti-mouse IgG antibody. Prior to mounting in anti-fade reagent, one drop of calcofluor white was applied on the slices for $10 \mathrm{~min}$ and washed three times with distilled water. Samples were examined using a Leica DMR fluorescence microscope (excitation filter 450-490 nm), coupled with a digital image capture system DFC500 (Leica).

\subsection{RNA extraction and $C D N A$ synthesis}

Plants were grown and etiolated as described above. After etiolation, $57 \mu \mathrm{M}$ of IAA, $57 \mu \mathrm{M}$ of NAA or media devoid of auxins was added to the wells to induce AR initiation. The whole seedlings were collected at the end of etiolation period (day zero) and at two, four, six, eight and ten days after exposure of seedlings to light and auxins (Fig. 1). Samples were frozen in liquid nitrogen and stored at $-80^{\circ} \mathrm{C}$. Total RNA was extracted using Concert Plant RNA Reagent (Invitrogen, Carlsbad, CA, USA), treated with DNAseI (EC number: 3.1.21.1, Invitrogen, Carlsbad, CA, USA) and the primary cDNA was synthesized from approximately $3 \mu \mathrm{g}$ of total RNA with oligo dT and reverse transcriptase (EC number: 2.7.7.49, M-MLV, Invitrogen, Carlsbad, CA, USA).

\subsection{Real Time RT-PCR}

The cDNA from each time point was diluted 100 times. Real-time quantitative reverse transcription-PCR was performed in an ABI 7500 Real-Time PCR System (Applied Biosystems). Primers were designed using Primer3 tool, version 0.4 (Rozen and Skaletsky, 2000) and are listed in Table 1. Reactions were done with SYBR green (1:10,000 from Molecular Probe) for detection, with total volume of $20 \mu \mathrm{l}$ and a cDNA fraction of 50\%. ACTIN (ACT) and ELONGATION FACTOR 1 (EF1) were selected as reference genes to normalize Real-Time RT-PCR data due to their stability under the experimental conditions, based on GeNorm and NormFinder softwares. A melting curve analysis was performed for every PCR program to ensure that the fluorescence signal was being generated by the desired amplification product. PCR efficiency was determined for each one of the amplifications using LinRegPCR software (Ramakers et al., 2003) and the average PCR efficiency for each gene was used in the calculations. To confirm the specificity of the amplified sequences, the amplicons were sequenced. The relative expression was calculated by the comparative $\Delta \Delta \mathrm{C}_{\mathrm{t}}$ method.

\subsection{Statistical analysis}

For root number and length experiments, 21 biological replicates were used. For Real Time RT-PCR experiments, three biological replicates were used with each replicate being composed of 15-22 plants. Each experiment was repeated three times. For immunohistological evaluation, 3 to 4 plants were used. Depending on normality assessment, statistical analyses used Welch's ANOVA followed by Dunnett's test (morphological data), ANOVA followed by Tukey test (gene expression within time points between treatments), or t-test (gene expression comparing each normalized expression data against the condition prior to the transfer to light and flooding and for two-treatment comparisons of morphological parameters in media with filtered or autoclaved auxins). In all cases, significance level was $P \leq 0.05$. The statistical software package IBM SPSS Statistics for Windows (Version 20.0) was used.

\section{Results}

\subsection{Effects of different auxin types on adventitious rooting}

In the absence of exogenous auxin, seedlings developed lower number of roots that displayed considerable length (Fig. 2). On day four, it was possible to observe increased number of AR with the application of exogenous IAA at $57 \mu \mathrm{M}$ and NAA at 40 and $57 \mu \mathrm{M}$, whereas no effect was seen with the use of 2,4-D at $500 \mathrm{nM}$ and $1 \mu \mathrm{M}$ (Fig. 2a). The application of 2 or $5 \mu \mathrm{M}$ of 2,4-D caused callus development instead of roots in all of the seedlings (Figs. 2 and 5). In general, root length was reduced in the presence of auxin when compared with the treatment without auxins on day four (Fig. 2b).

On day eight, distinct effects of auxin types on rooting became more evident. IAA caused an increase in number of ARs without significantly affecting root length. The response of adventitious rooting to NAA appeared to follow a dose dependent profile. When a concentration of $40 \mu \mathrm{M}$ of NAA was applied, ARs were induced at a higher efficiency than with IAA (Fig. 2a); however, roots remained short, as their extension was inhibited by NAA (Fig. 2 b). In presence of $2 \mu \mathrm{M}$ of NAA, the development of ARs did not differ from the control without auxins. When seedlings were treated with $1 \mu \mathrm{M}$ of $2,4-\mathrm{D}$, the number of ARs increased on day 8 (Fig. 2a). Root length was not affected by 2,4-D at $500 \mathrm{nM}$ or $1 \mu \mathrm{M}$ on day eight (Fig. 2b).

\subsection{IAA accumulation coincided with AR initiation tissues}

Immunolocalization was used to visualize IAA distribution in the

Table 1

Gene specific primers used for Real Time RT-PCR.

\begin{tabular}{|c|c|c|}
\hline Gene name & Forward Primer 5' $\rightarrow 3^{\prime}$ & Reverse Primer 5' $\rightarrow 3^{\prime}$ \\
\hline PIN1 & TGGTCCCTCATTTCCTTCAA & GGCAAAGCTGCCTGGATAAT \\
\hline PGP1 & TCTGGCGACTAGCTAAAATGAACTC & CCACAAATGACAGAGCCTACTGA \\
\hline$A U X 1$ & AAGGGCTTTGGCTAGATTGCC & CAAGAAGAGCACCGACAGCG \\
\hline CYP83B1/SUR2 & АCTCTTGACCCTAACCGCCСTAAAC & TGCAGCCGCCGTGTCAGT \\
\hline ARF6 uncleaved & CAAAGTTTAGCAGCTACCACGA & ACGTCGTTCTCTCGGTCAAC \\
\hline ARF8 uncleaved & TTTGCTATCGAAGGGTTGTTG & CATGGGTCATCACCAAGGA \\
\hline GH3.3 & ACAATTCCGCTCCACAGTTC & ACGAGTTCCTTGCTCTCCAA \\
\hline GH3.6 & CCTTGTTCCGTTTGATGCTT & CGTGTTACCGTTCAAGCAGA \\
\hline$A B P 1$ & ACTGCTGCAAGGCTGAAGTT & GGTTGTGCTTGCTTTTAGCC \\
\hline AGO1 & AAGGAGGTCGAGGAGGGTATGG & CAAATTGCTGAGCCAGAACAGTAGG \\
\hline SCARECROW & TCTGATCACGGTGGTGGAGCAAG & TCACCCAATCCATCTCCTAGCGC \\
\hline IAA28 & TCAAAGCCAAACCCCATTAG & TAAAGTTCTGGTCGGGGATG \\
\hline TIR1 & CCTAAACTGCAGCGCCTCT & GGTTGAAGCAAGCACCTCA \\
\hline CYCB1;1 & CTCAAAATCCCACGCTTCTTGTGG & CACGTCTACTACCTTTGGTTTCCC \\
\hline Actin & GCACCCTGTTCTTCTTACCG & AACCCTCGTAGATTGGCACA \\
\hline EF-1 & TGAGCACGCTCTTCTTGCTTTCA & GGTGGTGGCATCCATCTTGTTACA \\
\hline
\end{tabular}



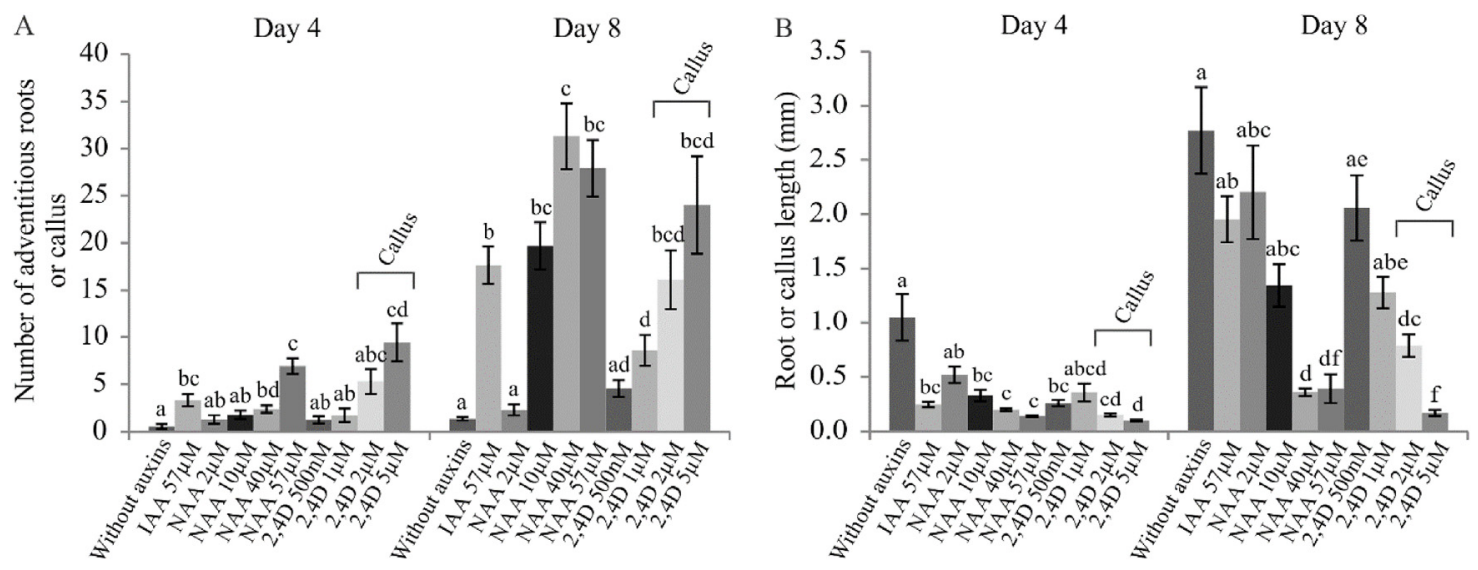

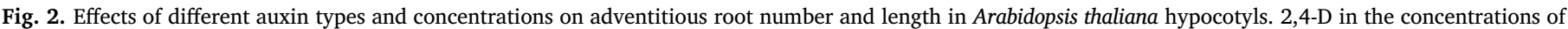

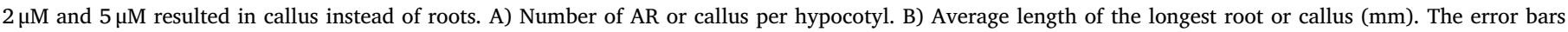
represent the standard error. Bars not sharing a letter are statistically different $(P \leq 0.05$, Welch's ANOVA followed by Dunnett's test).
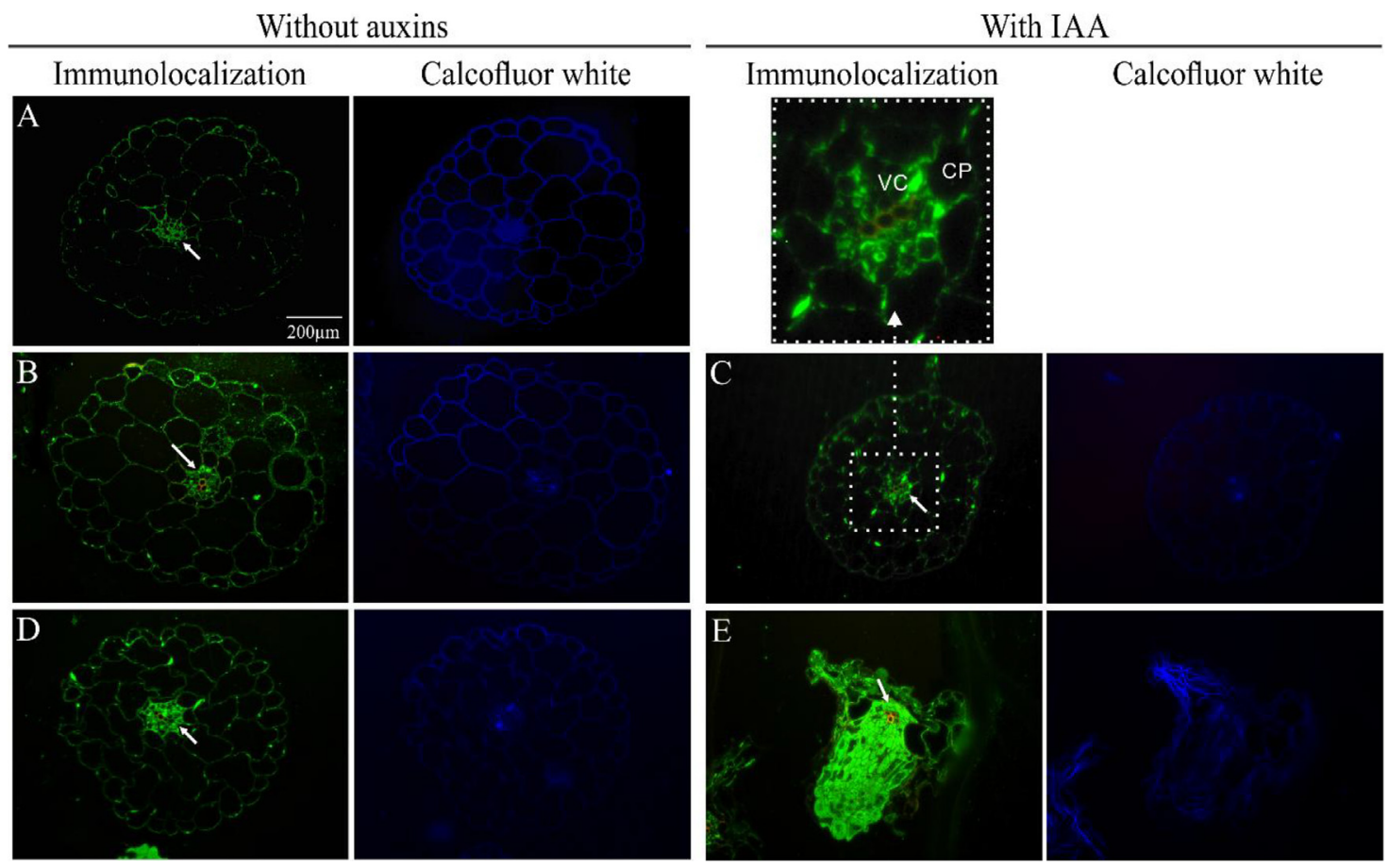

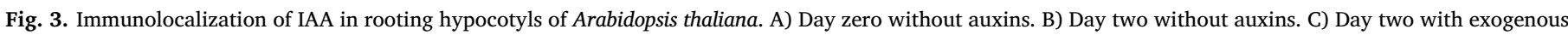

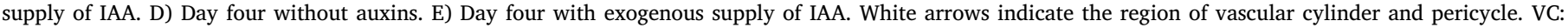
vascular cylinder; CP: cortical parenchyma.

hypocotyl of the seedlings, using anti-IAA antibodies. Fixed samples were treated first with anti-IAA monoclonal antibody and then with Alexa 488-conjugated goat anti-mouse IgG antibody. No fluorescence was detected when the slices were treated only with Alexa 488-conjugated goat anti-mouse IgG antibody (Supplementary Fig. 2). We checked the immunolocalization of IAA in seedlings treated or not with IAA. The images suggested a higher concentration of IAA in the regions of the vascular cylinder and the pericycle of the hypocotyl (Fig. 3). On day two with exogenous IAA and day four without supply of auxins higher accumulation of auxin was detected in points around the vascular cylinder, including the pericycle, where ARs are formed (Fig. 3c and d). On day four with external application of IAA it was possible to see the longitudinal section of an AR (Fig. 3e). There was intense accumulation signal of IAA along the root formed, the vascular cylinder and the pericycle of the hypocotyl.

\subsection{Expression of auxin related genes}

Gene expression analyses were conducted with seedlings treated with $57 \mu \mathrm{M}$ of IAA, $57 \mu \mathrm{M}$ of NAA or media devoid of auxins (control). Increased expression of PIN1, GH3.3 and GH3.6 was observed with both auxins (Fig. 4). SUR2, AUX1, ARF6, PGP1, ABP1 and AGO1 had similar profiles of higher expression with auxin (particularly with NAA), although not statistically significant compared to control (Supplementary Fig. 3). Expression of TIR1 decreased after flooding and transfer to light, then remaining constant over the periods analyzed. PGP1 expression followed an overall similar profile, except for NAA-treated seedlings at all times and IAA-treated at d6, 8 and 10 which kept the same level of expression compared to the initial condition in d0 (Supplementary Fig. 3). SCR showed variable and not significant differences when compared to control without auxin and had lower expression relative to pre-flooding and light condition, especially for auxin-treated seedlings 

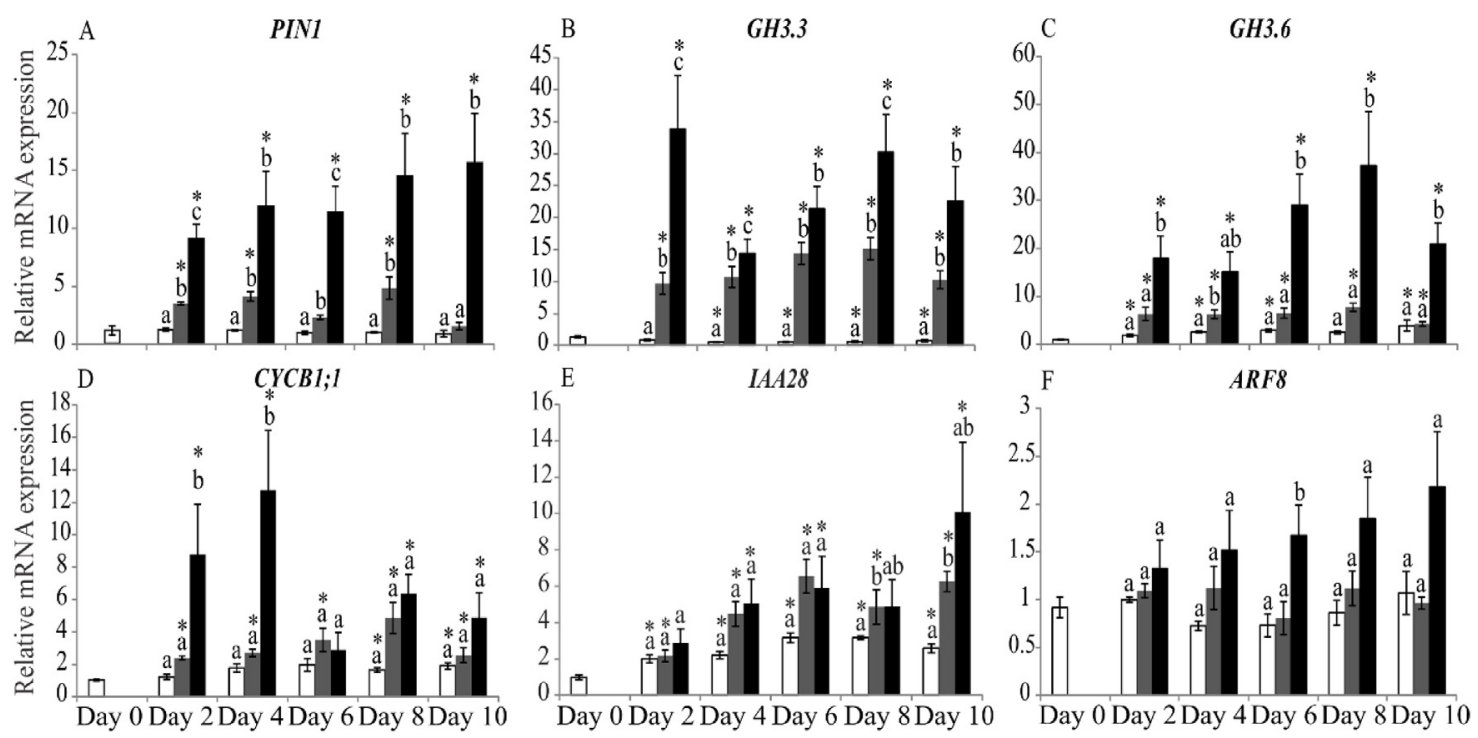

Fig. 4. Relative mRNA expression of auxin-related genes during adventitious rooting in etiolated seedlings without exogenous auxin (empty bars), with $57 \mu \mathrm{M}$ of IAA (grey bars) or $57 \mu \mathrm{M}$ of NAA (black bars). A. PINFORMED 1 (PIN1); B. GRETCHEN HAGEN 3.3 (GH3.3); C. GRETCHEN HAGEN 3.6 (GH3.6); D. CYCLIN B1;1 (CYCB1;1); E. AUX/IAA 28 (IAA28); F. AUXIN RESPONSE FACTOR 8 (ARF8). The error bars represent the standard error. The presence of an asterisk on top of a bar indicates significant difference compared to the respective expression on day zero ( $t$-test, $P \leq 0.05$ ). Bars sharing a letter are not significantly different within each time point (ANOVA followed by Tukey, $P \leq 0.05$ ).

in $\mathrm{d} 4$ and 6 (Supplementary Fig. 3). Compared to the initial pre-treatment condition, $A G O 1$ displayed increased expression after four days of treatment application, especially for control and IAA (Supplementary Fig. 3), $A R F 8, A R F 6$ and $A B P 1$ did not show significant changes in gene expression after flooding and transfer to light compared to pre-treatment stage (Fig. 4f, Supplementary Fig. 3).

PIN1 had increased expression as of $\mathrm{d} 2$ for both auxin treatments and progressively increased towards d10 in NAA treated seedlings, whereas IAA exposure yielded relatively stable increases with a reduction at d10 (Fig. 4a). NAA treatment raised auxin efflux transporter gene expression at $\mathrm{d} 2,6$ and 10. In contrast, no significant changes were recorded for $A U X 1$, except for the fact that it was less expressed at most time points after flooding and transfer to light (Supplementary Fig. 3).

GH3.3 and GH3.6 showed increased expression after flooding and transfer to light even without exogenous auxin supply (Fig. 4b and c). GH3.3 was upregulated both by NAA and IAA in relation to auxin-free media (Fig. 4b). Except for d4, when both auxins had the same effect, expression of GH3.6 was stimulated by NAA but not by IAA, suggesting a mostly NAA-specific response.

CYCB1 increased expression mainly on $\mathrm{d} 2$ and $\mathrm{d} 4$, but also $\mathrm{d} 8$ and $\mathrm{d} 10$, after flooding and transfer to light in the presence of exogenous auxins (Fig. 4d). After this period, CYCB1 expression decreased and there was no difference between media with or without auxins. Compared to the condition prior to flooding, higher expression in control seedlings was only observed in $\mathrm{d} 8$ and 10 , whereas auxin-treated seedlings had increased expression as of d2 (Fig. 4d). NAA-treated seedlings displayed strong induction in $\mathrm{d} 2$ and 4, surpassing the other treatments.

Surprisingly, IAA28 expression increased in the presence of IAA (Fig. 4e). This higher expression was significant in d8 and 10. NAA treatment, however, was not able to induce significantly higher expression of the same gene. There was an overall increased expression of this gene after flooding and transfer to light. In contrast, ARF8 expression was promoted in d6 upon NAA exposure relative to control and IAA treatment (Fig. 4f).

\section{Discussion}

In the absence of exogenous auxin, seedlings spontaneously developed relatively low numbers of roots, although these displayed considerable length, confirming the promptness of Arabidopsis hypocotyls to develop adventitious roots in this experimental system, which combines pre-etiolation and flooding to induce AR development (Correa et al., 2012). Both of these environmental factors may act as triggers for rooting by affecting variables such as phytohormonal balance, auxin transport and carbohydrate accumulation (Massoumi et al., 2017; Lu et al., 2017). Auxins stimulate adventitious rooting in the first stages of the process, but repress later developmental stages (De Klerk et al., 1999; Verstraeten et al., 2014). IAA was most effective in promoting adventitious rooting, probably because it stays in the tissues enough time to induce ARs, but not to inhibit elongation, since its conjugation as well as transport, may happen rapidly in the seedling (Kramer and Ackelsberg, 2015).

The application of NAA induced different responses depending on the concentrations used and the same was observed with 2,4-D. At 40 and $57 \mu \mathrm{M}$, NAA induced AR formation with comparable or higher efficiency than IAA, but root growth was markedly inhibited (Fig. 2). The effect of NAA in increasing the number, but reducing the length of the $\mathrm{AR}$ is probably related to its higher metabolic stability, being less oxidized, and capable of forming conjugates (De Klerk et al., 1999). In this sense, NAA has longer persistence than IAA in the tissues, probably becoming inhibitory to root elongation. In Eucalyptus globulus and E. saligna, $49.3 \mu \mathrm{M}$ of NAA promoted rooting percentage and number of roots in efficiency comparable to that of IAA, although yielding shorter roots (Fogaça and Fett-Neto, 2005). Considering root number and root length, the most effective concentration of NAA in the seedling flooding system was $10 \mu \mathrm{M}$, yielding responses similar to IAA (Fig. 2).

2,4-D was less efficient than IAA or NAA in promoting AR development. Among the concentrations tested of 2,4-D, $1 \mu \mathrm{M}$ was the most effective because, although the induction of roots was lower than that of IAA, it did not affect subsequent root growth (Fig. 2). Callus formation was observed with 2 or $5 \mu \mathrm{M}$ of 2,4-D (Figs. 2 and 5), what was also noticed by Verstraeten et al. (2013) in stem segments of $A$. thaliana with $10 \mu \mathrm{M}$ of 2,4-D and by Martínez-de la Cruz (2015) in maize. 2,4-D is poorly transported by auxin efflux proteins (Delbarre et al., 1996), 


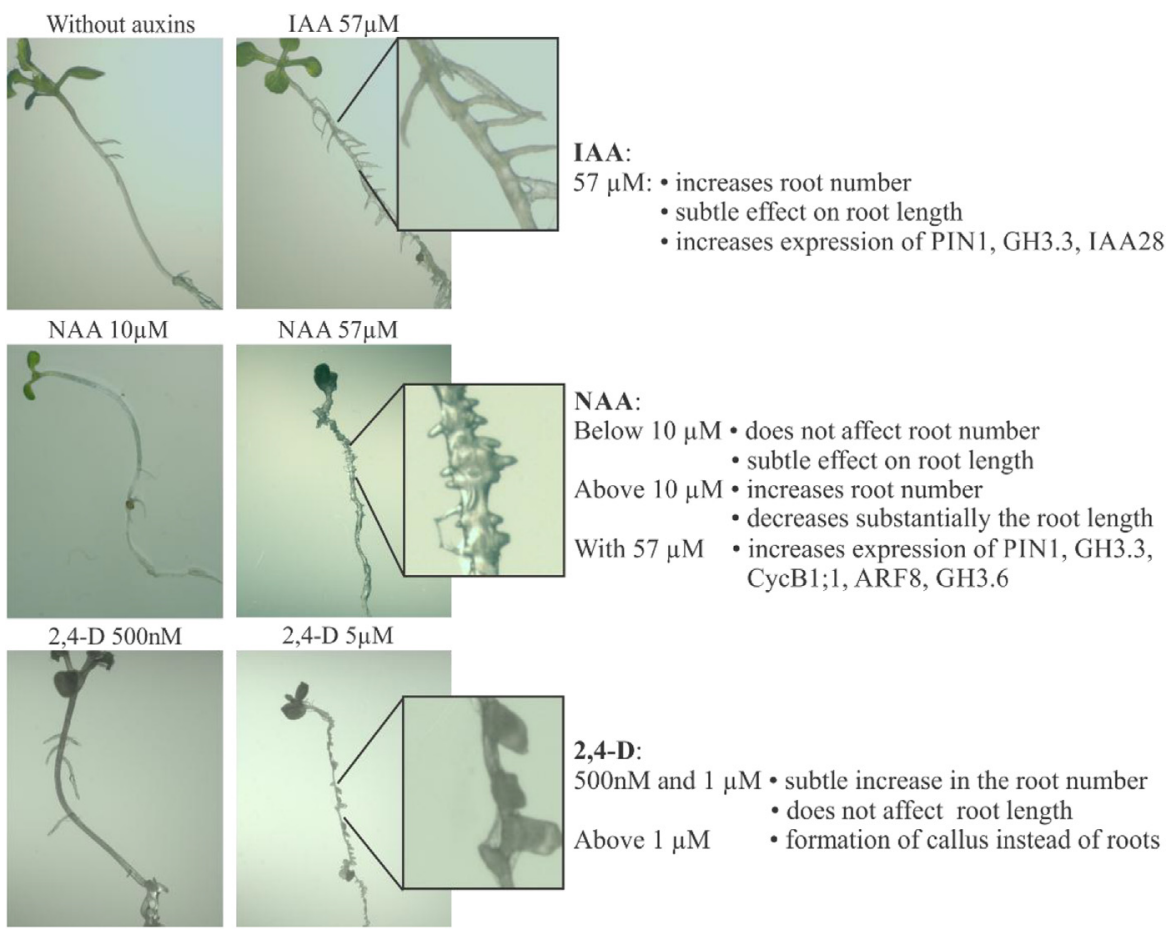

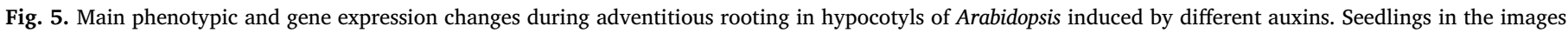
are in day 8 after the transfer to light and application of auxins by flooding.

which could cause an accumulation of this auxin type in the cells inhibiting AR initiation and leading to callus formation. In addition, 2,4-D has been shown to promote cell division, but not cell elongation in tobacco cells (Campanoni and Nick, 2005), which could contribute to callus formation. In fact it has been suggested that callus and de novo root organogenesis appear to share similar initiation mechanisms (Liu et al., 2014). Metabolic changes of 2,4-D in plants include reversible conjugation with amino acids or glucose and ring hydroxylation, which results in irreversible inactivation. Species that tolerate the herbicidal effect of this auxin are able to carry out ring hydroxylation (Peterson et al., 2016).

The immunolocalization of auxins allowed us to detect accumulation of auxin in the vascular cylinder and pericycle of the hypocotyl (Fig. 3), where a higher demand of this hormone is expected, since ARs are formed from these tissues in hypocotyls of flooded pre-etiolated seedlings (Correa et al., 2012). As expected, the signal of IAA-treated seedlings appears more intense than that of the control. Expression of PIN1 was observed by Della Rovere et al. (2013) in the same regions and was stimulated by exogenous auxin supply, suggesting that PIN1 might transport auxins from the hypocotyl vasculature to pericycle cells. This fact is also supported by PIN1 mRNA expression observed in etiolated seedlings (Fig. 4a), especially when IAA or NAA were provided. When the root is already formed (Fig. 3e) there is accumulation of IAA not only in the vascular cylinder and pericycle, but throughout the root. It is probable that this IAA pool is compartmentalized and/or conjugated, so as to allow for root elongation. This possibility is supported by the increased expression of GH3.3 in IAA treated seedlings (Fig. 4b). The IAA immunolocalization findings further support the key role of this phytohormone in root development of pre-etiolated hypocotyls under flooding.

PIN1, GH3.3, GH3.6, and CYCB 1;1 were upregulated by NAA (Fig. 4). PIN1 expression was also promoted by IAA treatment, but NAA had a higher effect at least in $\mathrm{d} 2$ and 6 (Fig. 4a). A similar profile of induction by both auxins was recorded for GH3.3, with stronger effects of NAA in d2, 4 and 8 (Fig. 4b). The significant induction of GH3.6 by NAA may indicate a need for tighter regulation of auxin concentration upon exposure to this auxin. Taken together, these differential increases in gene expression may explain the numerous shorter $\mathrm{AR}$ that are formed with NAA (at 40 and $57 \mu \mathrm{M}$ ) in relation to IAA $(57 \mu \mathrm{M})$ or in comparison to auxin-free media (Fig. 2). The effects of NAA in AR development may reflect not only its metabolic stability (De Klerk et al., 1999), but also the fact that it may enter cells by diffusion, being also recognized and transported by auxin efflux carriers (Delbarre et al., 1996).

Transcript levels of the auxin receptor TIR 1 decreased after flooding and transfer to light and the application of media with or without exogenous auxins, not changing over the subsequent period (Supplementary Fig. 3b). IAA and NAA compete efficiently for binding with TIR1, but IAA has a higher affinity for the receptor than NAA (Dharmasiri et al., 2005). However, it seems that receptor affinity differences did not affect gene expression in this case. ABP1 transcript accumulation also lacked statistically significant differences. Thus it was not possible to infer which receptor plays a major role in AR with this experimental system. Moreover, other members of the TIR1/AFBfamily of F-box proteins might also be involved in the adventitious rooting process. Molecular dynamics studies have shown that the interaction of NAA and ABP1 of Arabidopsis and maize is more stable than that of IAA with the same receptor (Da Costa et al., 2017), which might contribute to some of the relatively stronger induction of auxin-related gene expression and phenotypic responses by the former auxin.

PIN1 was induced by IAA and more intensely by NAA (Fig. 4a), while the relative mRNA expression of PGP1 was not significantly changed by the different treatments. PGP1 was apparently downregulated after flooding and transfer to light with IAA or in the absence of auxin (Supplementary Fig. 3e). PGP1 acts in the mediation of cellular efflux of IAA and NAA and its expression is auxin responsive; however, other auxin efflux proteins are involved in transport too (Geisler et al., 2005). PINs and PGPs have distinct transport mechanisms, playing different developmental roles that cooperate to mediate and enhance auxin transport (reviewed by Da Costa et al., 2013). The expression pattern of PIN1 suggests a major participation of PIN1 compared to $P G P 1$ in the adventitious rooting process promoted by auxins. Although expressed at lower levels than PIN1, PGP1 may play a role in the ARD process. The lack of reduction in PGP1 expression after applying 
irradiance and flooding relative to initial conditions in NAA treated seedlings (Supplementary Fig. 3) suggests a potential role in NAA cell removal for detoxification and homeostasis control. The influx carrier AUX1 did not show a clear pattern of expression and it was not induced by auxins, being downregulated after flooding and illumination mainly in control seedlings (Supplementary Fig. 3c). NAA enters the cells via passive diffusion and does not depend on influx carriers, whereas the control of NAA accumulation is mediated by efflux carriers (Delbarre et al., 1996), what could explain the prominent PIN1 mRNA levels of expression in the presence of this auxin type.

SUR2 is involved in the control of IAA and indole glucosinolate synthesis in Arabidopsis (Bak et al., 2001). The sur2 mutant overproduces IAA and displays a higher number of AR primordia (Delarue et al., 1998; Bak et al., 2001). Although SUR2 expression increased after flooding and light exposure, it was not significantly affected by auxin exposure, indicating coordinated auxin and secondary metabolite metabolism during rooting.

An interesting observation was the increased expression of the Aux/ IAA gene IAA28, in presence of exogenous IAA at the end of the rooting process (Fig. 4e). Previously, Rogg et al. (2001) found that auxin was not able to induce expression of IAA28; in fact, its mRNA levels decreased in response to IAA. This pattern was different from the ones observed for other Aux/IAA genes that are induced by auxins and show a biphasic dose response to IAA (Abel et al., 1995). In lateral roots, it was observed that the gain-of-function mutant iaa28-1 prevented lateral root formation and was resistant to auxin, suggesting that IAA28 could function in transcriptional repression of auxin-responsive genes involved in lateral root development (Rogg et al., 2001). Later, it was shown that lateral root founder cells are regulated by a GATA-type transcription factor, GATA23, which depends on the IAA28-ARF7ARF19 regulatory module (De Rybel et al., 2010). However, it is not clear how this regulation happens on AR formation and the role of IAA28 in this process requires further investigation. The expression profile of this gene may constitute another difference in the developmental pathways leading to lateral versus adventitious roots, a topic that has recently been reviewed (Verstraeten et al., 2014).

NAA treatment induced expression of ARF8 only in d6 (Fig. 4f). Expression of GH3.3 was induced by IAA and NAA, whereas GH3.6 was mainly induced by NAA (Fig. 4b and c). Gutierrez et al. (2012) proposed a model in which the auxin response factors ARF6 and ARF8 are positive regulators of the conjugation genes GH3.3, GH3.5 and GH3.6 that modulate jasmonic acid (JA) homeostasis and control AR initiation through the COI1 signaling pathway. JA is a considered a negative regulator of AR initiation in Arabidopsis and its conjugation to amino acids by GH3.3, GH3.5 and GH3.6 may regulate its concentration. In this regulation, AGO1 is also involved, by modulating ARF17 expression which negatively regulates expression of $\mathrm{GH} 3$ genes (Sorin et al., 2005). However, although AGO1 expression was significantly increased by NAA exposure in day 6, overall AGO1 and ARF6 mRNA expression was not significantly changed throughout the experiment and in the various treatments, suggesting that basal levels of expression were sufficient for regulating conjugation genes (Supplementary Fig. 3).

Steady state transcript levels of $S C R$ were lower in presence of exogenous auxins in relation to auxin-free medium in almost all of the periods analyzed (Supplementary Fig. 3h). Both in Pinus radiata D. Don and Castanea sativa Mill., an increase in mRNA expression of SCR-LIKE genes was observed in cuttings treated with exogenous auxin within the first $24 \mathrm{~h}$, indicating that this gene plays a role in the earlier stages of AR development, prior to cell divisions and the appearance of AR primordia (Sánchez et al., 2007). In the present work, we checked SCR expression after $48 \mathrm{~h}$ of auxin exposure, probably coinciding with decreased levels of SCR transcripts, as observed by Sánchez et al. (2007) in $P$. radiata and $C$. sativa. The reduced expression of $S C R$ seen in auxin treated seedlings relative to initial conditions (prior to irradiance and flooding application) may reflect a lower requirement of SCR in presence of exogenous auxin, since both cooperate in signaling to regulate
WOX5 expression in quiescent center delimitation and root meristem maintenance (Drisch and Stahl, 2015).

On the other hand, the mitotic cyclin $C Y C B 1 ; 1$ increased after transfer to light and flooding as of $\mathrm{d} 2$ in presence of auxins, but only at later time points for control seedlings (Fig. 4d), consistently with increased rooting in presence of auxins (Fig. 2a). This observation indicates that transcriptional programs of spontaneous adventitious rooting process are accelerated by exogenous auxin. The major peaks of mRNA level were observed in $\mathrm{d} 2$ and 4 in presence of NAA (Fig. 4d). Levels of mRNA also increased with IAA from $\mathrm{d} 2$ to 8 compared to initial conditions, although to a lower extent and without surpassing control levels. B-type cyclins play a role in the cell cycle and, more specifically, CYCB1;1 acts on G2 to M transition (Himanen et al., 2002). The increase in cyclin mRNA levels is closely related to the first cellular divisions that give rise to root primordia, indicating a correlation between induction of AR by auxin and cell cycle activation. Since the main phenotypic difference between NAA and IAA induced AR is root length (Fig. 2b), the higher early expression of CYCB1;1 in presence of NAA may also help explaining the development of numerous shorter roots formed with the synthetic auxin.

The effects of different auxins in adventitious root development and gene expression are summarized in Fig. 5. IAA increased the number of adventitious roots per explant without affecting root length, whereas NAA inhibited root growth at high concentrations and 2,4-D induced callusing. ARD was essentially marked by increased expression of PIN1, GH3.3 and GH3.6. Comparing the auxin conditions, some genes were predominantly or specifically induced with NAA (CYCB1;1, GH3.6, $A R F 8$ ), whereas IAA28 was IAA regulated. Overall, the magnitude of expression of induced genes was larger with NAA, followed by IAA, suggesting that auxin metabolic stability and transport rates may significantly change transcription profiles leading to ARD. IAA28 expression increased with ARD induced by IAA, unlike reports on lateral root development, possibly representing a differential feature between these two root developmental programs.

\section{Contributions}

Cibele Tesser da Costa helped designing the study, performed and analyzed the experiments, and drafted the manuscript.

Jorge Ernesto de Araujo Mariath and Marcos Letaif Gaeta helped with the immunolocalization experiments and their interpretation.

Remko Offringa devised some of the experiments and helped with data interpretation and discussion.

Arthur Germano Fett-Neto devised the experiments, helped with data interpretation and discussion, supervised the project and finalized the manuscript.

\section{Funding}

This work was supported by the Brazilian Agencies National Committee for Improvement of Higher Education Personnel (CAPES), National Council for Scientific and Technological Development (CNPq), and Rio Grande do Sul State Foundation for Research Support (Fapergs).

\section{Declarations of interest}

None.

\section{Appendix A. Supplementary data}

Supplementary data related to this article can be found at http://dx. doi.org/10.1016/j.plaphy.2018.03.022. 


\section{References}

Abel, S., Nguyen, M.D., Theologis, A., 1995. The PS-IAA4/5-like family of early auxininducible mRNAs in Arabidopsis thaliana. J. Mol. Biol. 251, 533-549.

Bak, S., Tax, F.E., Feldmann, K.E., Galbraith, D.W., Feyereisen, R., 2001. CYP83B1, a cytochrome P450 at the metabolic branch point in auxin and indole glucosinolate biosynthesis in Arabidopsis. Plant Cell 13, 101-111.

Campanoni, P., Nick, P., 2005. Auxin-dependent cell division and cell elongation: 1Naphthaleneacetic acid and 2,4-Dichlorophenoxyacetic acid activate different pathways. Plant Physiol. 137, 939-948.

Correa, L.R., Troleis, J., Mastroberti, A.A., Mariath, J.E., Fett-Neto, A.G., 2012. Distinct modes of adventitious rooting in Arabidopsis thaliana. Plant Biol. 14, 100-109.

Da Costa, C.T., De Almeida, M.R., Ruedell, C.M., Schwambach, J., Maraschin, F.S., FettNeto, A.G., 2013. When stress and development go hand in hand: main hormonal controls of adventitious rooting in cuttings. Front. Plant Sci. 4, 133. https://doi.org/ 10.3389/fpls.2013.00133.

Da Costa, C.T., Pedebos, C., Verli, H., Fett-Neto, A.G., 2017. The role of Zn2 +, dimerization and $\mathrm{N}$-glycosylation in the interaction of auxin-binding protein 1 (ABP1) with different auxins. Glycobiology 27, 1109-1119.

Della Rovere, F., Fattorini, L., D'Angeli, S., Veloccia, A., Falasca, G., Altamura, M.M. 2013. Auxin and cytokinin control formation of the quiescent centre in the adventitious root apex of Arabidopsis. Ann. Bot. 112, 1395-1407.

De Klerk, G.J., Van der Krieken, W., De Jong, J.C., 1999. The formation of adventitious roots: new concepts, new possibilities. In Vitro Cell. Dev. Biol. Plant 35, 189-199.

Delarue, M., Prinsen, E., Va, H., Caboche, M., Bellini, C., 1998. Sur2 mutations of Arabidopsis thaliana define a new locus involved in the control of auxin homeostasis. Plant J. 14 (5), 603-611.

Delbarre, A., Muller, P., Imhoff, V., Guern, J., 1996. Comparison of mechanisms controlling uptake and accumulation of 2,4-dichlorophenoxy acetic acid, naphthalene-1acetic acid, and indole-3-acetic acid in suspension-cultured tobacco cells. Planta 198, 532-541.

De Rybel, B., Vassileva, V., Parizot, B., Demeulenaere, M., Grunewald, W., Audenaert, D., Van Campenhout, J., Overvoorde, P., Jansen, L., Vanneste, S., Moller, B., Wilson, M., Holman, T., Van Isterdael, G., Brunoud, G., Vuylsteke, M., Vernoux, T., De Veylder, L., Inze, D., Weijers, D., Bennett, M.J., Beeckman, T., 2010. A novel aux/IAA28 signaling cascade activates GATA23-dependent specification of lateral root founder cell identity. Curr. Biol. 20, 1697-1706.

Dharmasiri, N., Dharmasiri, S., Estelle, M., 2005. The F-box protein TIR1 is an auxin receptor. Nature 435, 441-445.

Drisch, R.C., Stahl, Y., 2015. Function and regulation of transcription factors involved in root apical meristem and stem cell maintenance. Front. Plant Sci. 6, 505. http://dx. doi.org/10.3389/fpls.2015.00505.

Fogaça, C.M., Fett-Neto, A.G., 2005. Role of auxin and its modulators in the adventitious rooting of Eucalyptus species differing in recalcitrance. Plant Growth Regul. 45, 1-10.

Geisler, M., Blakeslee, J.J., Bouchard, R., Lee, O.R., Vincenzetti, V., Bandyopadhyay, A., Titapiwatanakun, B., Peer, W.A., Bailly, A., Richards, E.L., Ejendal, K.F.K., Smith, A.P., Baroux, C., Grossniklaus, U., Müller, A., Hrycyna, C.A., Dudler, R., Murphy, A.S., Martinoia, E., 2005. Cellular efflux of auxin catalyzed by the Arabidopsis MDR/ PGP transporter AtPGP1. Plant J. 44 (2), 179-194.

Geisler, M., Murphy, A.S., 2006. The ABC of auxin transport: the role of p-glycoproteins in plant development. FEBS Lett. 580 (4), 1094-1102.

Gutierrez, L., Mongelard, G., Flokova, K., Pacurar, D.I., Novak, O., Staswick, P., Kowalczyk, M., Pacurar, M., Demailly, H., Geiss, G., Bellini, C., 2012. Auxin controls Arabidopsis adventitious root initiation by regulating jasmonic acid homeostasis. Plant Cell 24, 2515-2527.

Himanen, K., Boucheron, E., Vanneste, S., De Almeida Engler, J., Inzé, D., Beeckman, T., 2002. Auxin-mediated cell cycle activation during early lateral root initiation. Plant Cell 14, 2339-2351.

Kamimoto, Y., Terasaka, K., Hamamoto, M., Takanashi, K., Fukuda, S., Shitan, N., Sugiyama, A., Suzuki, H., Shibata, D., Wang, B., Pollmann, S., Geisler, M., Yazaki, K., 2012. Arabidopsis ABCB21 is a facultative auxin importer/exporter regulated by cytoplasmic auxin concentration. Plant Cell Physiol. 53 (12), 2090-2100.

Kramer, E.M., Ackelsberg, E.M., 2015. Auxin metabolism rates and implications for plant development. Front. Plant Sci. 6, 150. https://doi.org/10.3389/fpls.2015.00150.

Liu, J., Sheng, L., Xu, Y., Li, J., Yang, Z., Huang, H., Xu, L., 2014. WOX11 and 12 are involved in the first-step cell fate transition during de novo root organogenesis in Arabidopsis. Plant Cell 26, 1081-1093.

Lu, N., Dai, L., Luo, Z., Wang, S., Wen, Y., Duan, H., Hou, R., Sun, Y., Li, Y., 2017. Characterization of the transcriptome of tetraploid black locust cuttings in response to etiolation. Genes 8, 345. http://dx.doi.org/10.3390/genes8120345.

Maraschin, F.S., Memelink, J., Offringa, R., 2009. Auxin- induced, SCF(TIR1)-mediated poly- ubiquitination marks AUX/IAA proteins for degradation. Plant J. 59, 100-109.

Martínez-de la Cruz, E., García-Ramírez, E., Vázquez-Ramos, J.M., de la Cruz, H.R., López-Bucio, J., 2015. Auxins differentially regulate root system architecture and cell cycle protein levels in maize seedlings. J. Plant Physiol. 176, 147-156.

Massoumi, M., Krens, F.A., Visser, R.G.F., De Klerk, G.-W.M., 2017. Etiolation and flooding of donor plants enhance the capability of Arabidopsis explants to root. Plant Cell Tissue Organ Cult. 130, 531-541.

Morris, D.A., Friml, J., Zažímalová, E., 2010. The transport of auxins. In: Davies, P.J. (Ed.), Plant Hormones. Springer Netherlands, Netherlands, pp. 451-484.

Nodzyński, T., Vanneste, S., Friml, J., 2012. Endocytic trafficking of PIN proteins and auxin transport. In: Šamaj, J. (Ed.), Endocytosis in Plants. Springer Berlin Heidelberg, Berlin, pp. 165-183.

Péret, B., Swarup, K., Ferguson, A., Seth, M., Yang, Y., Dhondt, S., James, N., Casimiro, I., Perry, P., Syed, A., Yang, H., Reemmer, J., Venison, E., Howells, C., Perez-Amador, M.A., Yun, J., Alonso, J., Beemster, G.T., Laplaze, L., Murphy, A., Bennett, M.J., Nielsen, E., Swarup, R., 2012. AUX/LAX genes encode a family of auxin influx transporters that perform distinct functions during Arabidopsis development. Plant Cell 24 (7), 2874-2885.

Pérez-Hidalgo, L., Moreno, S., Martín-Castellanos, C., 2008. Modified cell cycle regulation in meiosis. In: Egel, R., Lankenau, D.-H. (Eds.), Recombination and Meiosis. Springer Berlin/Heidelberg, Berlin, pp. 307-353.

Peterson, M.A., McMaster, S.A., Riechers, D.E., Skelton, J., Stahlman, P.W., 2016. 2,4-D past, present, and future: a review. Weed Technol. 30 (2), 303-345.

Ramakers, C., Ruijter, J.M., Deprez, R.H.L., Moorman, A.F., 2003. Assumption-free analysis of quantitative real-time polymerase chain reaction (PCR) data. Neurosci. Lett. 339 (1), 62-66.

Rogg, L.E., Lasswell, J., Bartel, B., 2001. A gain-of-function mutation in IAA28 suppresses lateral root development. Plant Cell 13 (3), 465-480.

Rozen, S., Skaletsky, H.J., 2000. Primer3 on the WWW for general users and for biologist programmers. In: Krawetz, S., Misener, S. (Eds.), Bioinformatics Methods and Protocols: Methods in Molecular Biology. Humana Press, Totowa, NJ, USA, pp. 365-386.

Sánchez, C., Vielba, J.M., Ferro, E., Covelo, G., Sole, A., Abarca, D., de Mier, B.S., DiazSala, C., 2007. Two SCARECROW-LIKE genes are induced in response to exogenous auxin in rooting-competent cuttings of distantly related forest species. Tree Physiol. $27,1459-1470$.

Sorin, C., Bussell, J.D., Camus, I., Ljung, K., Kowalkzyc, N., Geiss, G., McKhann, H., Garcion, C., Vaucheret, H., Sandberg, G., Bellini, C., 2005. Auxin and light control of adventitious rooting in Arabidopsis require ARGONAUTE1. Plant Cell 17, 1343-1359.

Strader, L.C., Culler, A.H., Cohen, J.D., Bartel, B., 2010. Conversion of endogenous indole3-butyric acid to indole-3-acetic acid drives cell expansion in Arabidopsis seedlings. Plant Physiol. 153, 1577-1586.

Strader, L.C., Zhao, Y., 2016. Auxin perception and downstream events. Curr. Opin. Plant Biol. 33, 8-14.

Verstraeten, I., Beeckman, T., Geelen, D., 2013. Adventitious root induction in Arabidopsis thaliana as a model for in vitro root organogenesis. In: De Smet, I. (Ed.), Plant Organogenesis: Methods and Protocols. Springer Science + Business Media, New York, pp. 159-175.

Verstraeten, I., Schotte, S., Geelen, D., 2014. Hypocotyl adventitious root organogenesis differs from lateral root development. Front. Plant Sci. 5, 1-13. https://doi.org/10. 3389/fpls.2014.00495.

Yin, K., Ueda, M., Takagi, H., Kajihara, T., Sugamata Aki, S., Nobusawa, T., Umeda-Hara, C., Umeda, M., 2014. A dual-color marker system for in vivo visualization of cell cycle progression in Arabidopsis. Plant J. 80 (3), 541-555. 